\title{
Research and Application of Physician Evaluation Index Based on Rough Set Theory
}

\author{
An Song ${ }^{4}$, YunHua , Zhang ${ }^{1}$ \\ School of Zhejiang Sci-Tech University, Hangzhou 310018, China \\ 849953550@qq.com
}

\begin{abstract}
Keywords: comprehensive evaluation; evaluation index; large data analysis; rough set theory; attribute reduction
\end{abstract}

\begin{abstract}
Physician evaluation is an indispensable part of the resident physicians in their growth process, which is an important means of assessing the physician's comprehensive ability. In the comprehensive evaluation and decision analysis, the correct selection of evaluation indicators is one of the key links, directly affecting the final results of evaluation and decision-making. The traditional evaluation indexes of physicians mainly adopt questionnaire survey method, princip al component analysis method and expert advice and so on. This is more subjective and has a great impact on physicians' evaluation. In order to improve traditional physician evaluation accuracy is insufficient, this article embarks from the physicians the large data in the process of growth, the basic data to deal with the noise, and then after the pretreatment of data mining. It is mainly based on the attribute reduction algorithm in rough set theory to study the evaluation indexes of physicians, and evaluate the comprehensive abilities of doctors scientifically and objectively. The experiment shows that this method can improve the accuracy of the comprehensive evaluation of physicians, so it is effective and feasible, and can be popularized in practical application.
\end{abstract}

\section{Introduction}

In the second half of the 20th century, the traditional "one-stop" school medical education system was challenged and replaced by a medical lifelong education system. This kind of medical education system consists of the organic combination of school medical education, after graduating medical education and continuing medical education three parts. This provides an organizational and institutional guarantee for the selection, training and renewal of medical professionals. Nowadays, market competition is the competition of talents. In the management, hospitals promote the cultivation of talents, which is to realize the improvement of hospital competitiveness through the cultivation of talents. In order to more effectively promote the growth of talents and promote the development of the hospitals, it is urgent to improve the work efficiency and improve the evaluation system of doctors.

With the development of society, people's quality of life requirements and the importance of the value of life are constantly improving, but also began to re-evaluate the new look of medical development, the resident of the scientific and effective evaluation of more and more attention. The doctor's regular assessment is to further examine the physician's ability to practice, to strengthen the management of the physician team, standardize the practice of physicians, improve the quality of physicians, ensure the quality of medical care and medical security, promote safe hospital construction, create a harmonious doctor-patient relationship very important meaning.

The physician's comprehensive evaluation is often a multi-objective complex problem that includes both qualitative and quantitative indicators. At present, a variety of comprehensive evaluation methods such as questionnaire survey method, expert suggestion method, principal component analysis method, gray relational degree evaluation and fuzzy comprehensive evaluation are adopted. However, the above evaluation method has certain limitations in the selection of the evaluation index and the determination of the weight, and can't obtain the evaluation result accurately and objectively. 
In this paper, the data is preprocessed by the large amount of basic data in the process of physician growth. Based on the attribute reduction algorithm in rough set theory, the physician evaluation index and scientific evaluation physician's comprehensive ability are studied.

\section{Organization of the Text}

\subsection{Algorithm introduction}

Rough set theory is a new mathematical tool for dealing with fuzzy and uncertain knowledge. The main idea is to derive the problem through the reduction of knowledge and the irrelevant or unimportant attribute under the premise of keeping the classification ability unchanged Decision-making or classification rules. The most important feature of it is that it does not need to provide any prior knowledge of the data set needed to solve the problem. Only the classification of the measured data itself can be used to excavate the implied knowledge and reveal the underlying data. It can handle incomplete data information, and can retain the key data in the premise of the data to simplify the knowledge of the minimum expression of knowledge, and can assess the dependencies between the data, from the empirical data to obtain easy to verify the classification Rules that are conducive to intelligent control. On the one hand, it can deal with incomplete data, which can keep key data information in under the premise of the data reduction and the minimum expression of knowledge, on the other hand, it can evaluate the dependent relationships between data, which is obtained from the empirical data is easy to verify the classification rules, thus is advantageous to the intelligent control. At present, rough set theory has been successfully applied in machine learning, decision analysis, process control , data mining and other fields.

2.2.Algorithm definition

Let $\mathrm{S}$ be an information system, which is called a decision table, and a four-tuple can be used to denote $S=(U, A, V, f)$, where $\mathrm{U}$ is the universe and $U \neq \emptyset ; A=C \cup D, \mathrm{C}$, and D represent the set of conditional attributes and the set of decision attributes, respectively, and $C \cap D=\emptyset, \mathrm{V}$ represents the set of values, $V=\bigcup_{a \in A} V_{a}, V_{a}$ represents the range of a; Function $f: U \times A \rightarrow V$, for $\forall x \in U, a \in A$, there $f(x, a) \in V$.

Definition 1 : For any attribute subset $R \subseteq \mathrm{C}$, If

$$
\operatorname{IND}(R)=\left\{\left(x_{i}, x_{j}\right) \in(U \times U) \mid \forall a \in R, f\left(x_{i}, a\right)=f\left(x_{j}, a\right)\right\},
$$

then $\mathrm{R}$ is an indistinguishable relation of decision table $\mathrm{S}$, and all equivalence classes of equivalence relation IND $(\mathrm{R})$ are denoted by $\mathrm{U} / \mathrm{R}$.

Definition 2 : The entropy of attribute set $\mathrm{R}$,

$$
H\left(X_{i}\right)=-\sum_{i=1}^{n} P\left(X_{i}\right) \log P\left(X_{i}\right), P\left(X_{i}\right)=\left|X_{i}\right| /|U| .
$$

This describes the uncertainty of the knowledge contained in the rough set.

Definition 3 : In the decision system $\mathrm{S}$, the conditional entropy of the decision set $\mathrm{D}$ relative to the conditional attribute R set :

$$
\begin{array}{r}
H(D \mid R)=-\sum_{i=1}^{n} P\left(X_{i}\right) \sum_{j=1}^{m} P\left(Y_{j} \mid X_{i}\right) \log P\left(Y_{j} \mid X_{i}\right), \\
p\left(Y_{j} \mid X_{i}\right)=\frac{\left|Y_{j} \cap X_{i}\right|}{\left|X_{i}\right|} i=1,2, \ldots, n, j=1,2, \ldots, m
\end{array}
$$

The higher the conditional entropy, the higher the uncertainty of the conditional attribute relative to the decision attribute, that is, the smaller the number of objects that can be allocated to the U/D class according to the partition U/R.

Definition 4 : the mutual information I (R; D) of the attribute set $\mathrm{R}$ with respect to the decision attribute $\mathrm{D}$ is defined as :

$$
I(R ; D)=H(D)-H(D \mid R)
$$

Definition 5 : Let $R \subseteq \mathrm{C}$, and $I(R ; D)=I(C ; D)$, If for any $\mathrm{r} \in \mathrm{R}$, there are $I(R-$ $\{r\} ; D)<I(R ; D)$, We say $\mathrm{R}$ is a property reduction of the condition attribute set $\mathrm{C}$ with respect to decision $\mathrm{D}$. 


\subsection{Experiment procedure}

The minimum attribute reduction of the evaluation index in the physician evaluation system refers to a reduction of the least number of attributes in the attribute reduction of the physician evaluation index attribute C. Therefore, the process of solving the best evaluation index and weight of the physician is as follows:

(1) The information of the physician evaluation system of the hospital was collected, and the statistical comparison and analysis were carried out. Then, a unified and reasonable physician evaluation index attribute and a weight proportion were collected.

(2) The data is recorded from a hospital database, and the data is preprocessed to remove some incomplete and invalid data. The data is then quantified.

(3) According to the similar relationship and similar formulas defined above, the pre-processed data is attributed to the attribute reduction, and the above work is repeated to obtain a best reduction attribute.

(4) Comparison and analysis of the evaluation index of doctors in this paper is related to the indexes in the evaluation system of doctors in the original hospital, and the corresponding conclusions are obtained.

(5) Again select several hospital database of Chinese medicine practitioners to grow the record data, repeat the above work. Draw the corresponding conclusion.

2.4.Experimental results and analysis

In some hospital databases, this paper extracts the data of the growth process of some doctors. Based on the attribute reduction algorithm in rough set theory, the condition attribute of the evaluation index of physicians is reduced, and the results are as follows:

\begin{tabular}{|l|l|c|c|c|}
\hline $\begin{array}{l}\text { Hospital } \\
\text { Information }\end{array}$ & Samples & $\begin{array}{l}\text { Original Evalu- } \\
\text { a tion indicators }\end{array}$ & $\begin{array}{l}\text { Evaluation indicators } \\
\text { in this method }\end{array}$ & $\begin{array}{l}\text { Indicator weight } \\
\text { changes(\%) }\end{array}$ \\
\hline Hospital A & 545 & 31 & 28 & $5.3 \%$ \\
\hline Hospital B & 687 & 29 & 28 & $15 \%$ \\
\hline Hospital C & 655 & 40 & 32 & $8.3 \%$ \\
\hline Hospital C & 786 & 35 & 31 & $6.4 \%$ \\
\hline Hospital E & 486 & 33 & 30 & \\
\hline
\end{tabular}

The experimental results shows that most of the hospital doctor evaluation system basically sound, but the doctor evaluation index number and based on the attribute reduction in data mining to calculate the number of differences. Among them, especially $\mathrm{C}$ hospital doctors and the serious problem of evaluation system of evaluation index system of the old physician is not complete, there are repeated many properties, and weight distribution is not reasonable, comprehensive evaluation on the accuracy of the serious influence physicians. Based on the attribute reduction of data mining, the evaluation index of physicians is basically stable, which improves the scientific nature and accuracy of doctors' evaluation, which is beneficial to the healthy development of doctors.

\section{Conclusion}

The selection quality of the evaluation indexes directly affects the scientific and effectiveness of the comprehensive evaluation of physicians. Compared with the traditional method, through the mining of a large number of basic data in the process of physician growth, the attribute reduction algorithm based on rough set theory is used to analyze and determine the physician evaluation index, which improves the accuracy of physician comprehensive evaluation. The perfect evaluation system provides a theoretical basis for the hospital management department to comprehensively evaluate the physician's ability to work. Only by a scientific evaluation system for each physician's work to 
make a correct evaluation can we make everyone better in the face of their status quo and continue to improve, finally further raise the health care's quality and service level.

\section{References}

[1]Pawlak Z.Rough Sets:Theoretical Aspects of Reasoning about Data[M].Boston, London,Dordrecht:KluwerAcademic Publishers, 1991.

[2] T.-P. Hong, L.-H. Tseng, and S.-L. Wang, "Learning rules from incomplete training examples by rough sets,” Exp. Syst. Appl., vol. 22, no. 4, pp. 285-293, May 2002.

[3] M. Salamo and M. Lopez-Sanchez, "Rough set based approaches to feature selection for case-based reasoning classifiers," Pattern Recognit. Lett.,vol. 32, no. 2, pp. 280-292, Jan. 2011.

[4]D. Tian, X.-J. Zeng, and J. Keane, "Core-generating approximate minimum entropy discretization for rough set feature selection in pattern classification," Int. J. Approx. Reason., vol. 52, no. 6, pp. 863-880, Sep.2011.

[5] F. Witlox and H. Tindemans, "The application of rough sets analysis in activity-based modelling. Opportunities and constraints,” Exp. Syst. Appl.,vol. 27, no. 4, pp. 585-592, Nov. 2004.

[6] P. J. Lingras and Y. Y. Yao, “Data mining using extensions of the rough set model,” J. Amer. Soc. Inf. Sci., vol. 49, no. 5, pp. 415-422, 1998.

[7] C.-S. Son, Y.-N. Kim, H.-S. Kim, H.-S. Park, and M.-S. Kim, “Decision-making model for early diagnosis of congestive heart failure using roughset and decision tree approaches," J. Biomed. Informat., vol. 45, no. 5,pp. 999-1008, Oct. 2012.

[8] JiYe Liang , KaiShe Qu , ZongBen XU. Attribute reduction of information system[J]. System Engineering Theory and Practice,2001,12(3).

[9] Jelonek J, Krawiec K, Slowinski R. Rough set reduction of attributes and their domains for neural networks[ J]. Computation Intelligence, 1995, 11(2):339-347.

[10]Smyth P, Goodman R M. An information theoretic approach to rule induction from databases[ J]. IEEE Trans. Knowledge Discovery Data Mining, 1992(4):301-316. 